\title{
SGEM Hot Off the Press: Management of bronchiolitis in community hospitals
}

\author{
Josh J. Wang, MD*; Rohit Mohindra, $\mathrm{MD}^{\dagger}$; Ken Milne, $\mathrm{MD}^{\ddagger \S}$; Anthony Crocco, MD"; Chris Bond, MD"
}

Keywords: bronchiolitis, medical education, knowledge translation

As part of the Canadian Journal of Emergency Medicine's (CJEM) developing social media strategy, ${ }^{1}$ we are collaborating with the Skeptics' Guide to Emergency Medicine (SGEM) to summarize and critically appraise the current emergency medicine literature using evidence-based medicine principles. In the "Hot Off the Press" (HOP) series, we select original research manuscripts published in CJEM to be summarized and critically appraised on the SGEM website/podcast and discussed by the study authors and the online EM community. A similar collaboration is underway between the SGEM and Academic Emergency Medicine.

What follows is a summary of the selected article and the immediate post-publication critical appraisal from the SGEM podcast, as well as an overview of the subsequent discussion from the SGEM blog and other social media. Through this series, we hope to enhance the value, accessibility, and application of important, clinically-relevant EM research. In the sixth SGEM HOP hosted collaboratively with CJEM, we discuss Plint and colleagues' report ${ }^{2}$ on the treatment of bronchiolitis in Canadian community emergency departments.

\section{BACKGROUND}

Bronchiolitis is a common pediatric respiratory infection that causes inflammation and mucous plugging of the lower airways, typically in children 3 to 6 months of age. It is a clinical diagnosis that usually requires no laboratory or radiographic investigations. To the frustration of many pediatric and emergency care providers, the benefits of bronchodilators, corticosteroids, and antibiotics in bronchiolitis remain unproven, and there remains significant variation in the treatment of this disease. ${ }^{3-6}$

\section{SUMMARY}

Plint and colleagues sought to characterize practice patterns for the treatment of bronchiolitis in Canadian community hospitals. ${ }^{2}$ Their multicentre, retrospective cohort study recruited patients 12 months of age and younger with bronchiolitis between 2005 and 2007 from Ontario emergency departments (EDs) belonging to hospitals that were not primarily referral centres. The investigators excluded patients with a previous diagnosis of asthma and treatment of a cough, wheezing, or both with bronchodilators, steroids, or a combination of these medications during any previous visit. A trained analyst extracted the data by chart review.

The primary outcome measure was patient disposition (admission, discharge, and hospital transfer), and the secondary outcome measures included the use of medications, ancillary testing, and return to ED in the 21 days subsequent to the index ED visit.

\section{KEY RESULTS}

The investigators analyzed the records of 543 eligible patients from 28 hospitals and found that $30 \%$ of patients were admitted to the community hospital, and $3 \%$ of patients were transferred to a tertiary care centre. Seven percent had a return ED visit within 21 days. Further,

From the *McGill FRCP Emergency Medicine Residency Program, Montreal, QC; †Department of Emergency Medicine, Sir Mortimer B. Davis Jewish General Hospital, Montreal, QC; ‡Department of Emergency Medicine, Western University, London, ON; §Emergency Medicine, South Huron Hospital, Exeter, PE; IDepartment of Pediatrics, McMaster University, Hamilton, ON; and IDepartment of Emergency Medicine, University of Calgary, Calgary, AB.

Correspondence to: Josh J. Wang, 1001 Boulevard Décarie, Chambre/Room CS16237.1, Montreal, QC H4A 3J1; Email: joshwang.md@gmail.com 


\begin{tabular}{|c|c|c|c|}
\hline \multicolumn{4}{|c|}{ Patients in ED $(N=543)$} \\
\hline Investigation & n (\%) & $95 \% \mathrm{Cl}(\%)$ & $10 R^{*}$ \\
\hline Chest x-ray & $296(54.5)$ & $46.2-62.9$ & $30.9-60.7$ \\
\hline Nasal viral studies & $123(22.7)$ & $10.3-34.9$ & $1.0-28.0$ \\
\hline Any blood work & $37(6.8)$ & 3.8-9.8 & $0-8.5$ \\
\hline $\mathrm{CBC}$ & $28(5.2)$ & & \\
\hline Electrolytes & $9(1.7)$ & & \\
\hline Blood gas & $9(1.7)$ & & \\
\hline Blood culture & $4(0.7)$ & & \\
\hline Any urine study & $16(3.0)$ & $1.3-4.5$ & $0-3.2$ \\
\hline
\end{tabular}

$80 \%$ of the patients were given bronchodilators, $31 \%$ were provided corticosteroids, and 5\% received antibiotics. Of the 378 patients discharged from the ED, $45 \%$ were given prescriptions for bronchodilators, $24 \%$ for corticosteroids, and $13 \%$ for antibiotics. The most common investigations these patients underwent were chest radiographs, followed by nasal viral studies, blood work, and urine studies (Table 1).

\section{QUALITY ASSESSMENT}

The authors conducted a large, retrospective, and multicentre cohort study with patient-level data. The participation rate was $37 \%$ of eligible hospitals, with each institution acquiring research ethics approval. The study captured a sample of hospitals with large differences in annual ED visits, annual visits for bronchiolitis, and distance to nearest referral centre. Primary and secondary outcomes were clearly defined in the study methods and were both patient and clinician oriented.

Eligible patients whose medical charting matched the study definition of bronchiolitis without the aforementioned exclusion criteria were carefully recruited from those with a discharge diagnosis of "bronchiolitis," "wheezing," "respiratory syncytial virus" (RSV), "asthma," and "reactive airways." Despite hospital participation being non-random, selection bias was likely minimized through this recruitment method. However, the authors could have obtained a larger study population by expanding the pool of eligible patients to include a broader set of discharge diagnoses such as "pneumonia" and "fever."
The quality and internal validity of the study data were high because the same trained data analyst visited each study site to conduct chart reviews. Double extraction by a separate data analyst and a formal assessment of inter-rater reliability in a subset of these charts for inclusion and exclusion criteria and outcome data would have improved reliability. However, time and resource constraints may have precluded this additional step.

This study identified broad trends in the admission, transfer, investigation, and treatment rates for bronchiolitis patients in community EDs, but the wide interquartile range and $95 \%$ confidence intervals indicate poor precision. The small sample size per recruitment site, heterogeneous clinical approach to bronchiolitis, and non-normal distribution of outcome data all likely contributed to the high measures of variance.

A large number of the study patients received bronchodilators, corticosteroids, and antibiotics. Although the authors concluded from these results that patients with bronchiolitis in community hospitals received medications that had little evidence of benefit, the use of beta- 2 agonists in this study was consistent with contemporary bronchiolitis practice patterns. The 2006 American Association of Pediatrics (AAP) bronchiolitis guidelines published halfway through the data collection period included a recommendation for a short trial of inhaled bronchodilators. ${ }^{7}$ In addition, the way in which antibiotic use was reported in this study makes it difficult to identify whether the indication was an uncomplicated bronchiolitis or a concurrent bacterial infection that exists in a significant proportion of these patients. ${ }^{8,9}$ Further, there is evidence that resource utilization and practice variation in bronchiolitis has decreased in the 10 years since the study data were collected. $^{10}$ Nonetheless, we agree with the authors that these results suggest overtreatment and over-investigation of bronchiolitis in community hospitals but believe that these conclusions must be tempered by the time elapsed since the original data was collected, community physicians' relative comfort with pediatric respiratory complaints, ${ }^{11}$ and the margin for diagnostic error in rural settings with limited specialist support and difficult patient follow-up.

\section{TAKE-TO-WORK POINTS}

Bronchiolitis management in clinical practice remains fraught with interventions that show no evidence of benefit. ${ }^{10}$ The study by Plint and colleagues on practice 
patterns in Canadian community hospitals between 2005 and 2007 suggests a tendency for clinicians to administer medications and order tests beyond what was recommended by contemporary practice guidelines. Knowledge translation efforts will play a role in minimizing the time between future advances in bronchiolitis management and their uptake in community practice.

\section{METHODOLOGY OF THE ANALYSIS OF THE SOCIAL MEDIA RESPONSE}

The blog post and podcast were released on December 18,2016 , and the social media discussion continued for one week up to December 25, 2016. Listeners and viewers were invited to comment on the article through the podcast audio, blog post text, Twitter, and Facebook. The social media responses found in the Skeptics' Guide to Emergency Medicine (SGEM) blog comments, on the SGEM Facebook page, and on Twitter (directed using the \#SGEMHOP hashtag) between December 18, 2006, and December 25, 2016, were reviewed by the authorship team. JW and KM compiled and reviewed all aforementioned social media commentary and identified and conducted a thematic analysis of tweets and posts related to the CPOE SGEM podcast and blog post.

Multiple metrics of dissemination were further tracked by the SGEM HOP team for analysis:

- Blog post page views were monitored using the Jet Pack plugin by Wordpress.com (available from: https://wordpress.org/plugins/jetpack/).

- Facebook "reach" analytics were provided by Facebook and represented the number of users who saw the original SGEM Facebook post on their own newsfeeds. ${ }^{12}$

- Twitter impressions (the number of users whose newsfeeds contained a tweet featuring the \#SGEMHOP hashtag) were tracked using Symplur, a software program that monitors health care-related Twitter conversations. ${ }^{13}$

- Tweets not containing the hashtag were not tracked by Symplur. The number of impressions was calculated by taking the number of tweets per Twitter user using the \#SGEMHOP hashtag and multiplying it by the number of followers each participant had.

- The Altmetric score is a proprietary, standardized tool that tracks the disseminative impact of research articles in social media forums (e.g., Facebook and Twitter) and on blogs, podcasts, and news outlets. ${ }^{14}$ The Altmetrics of the featured article by Plint and colleagues were compared to all other articles published in CFEM and all published research analyzed by Altmetric.

\section{RESULTS OF THE SOCIAL MEDIA RESPONSE}

Between December 18 and December 25, 2016, this SGEM HOP had an Altmetric Attention Score of 48, ranking ninth amongst all CFEM publications and amongst the top fifth percentile of all articles ever tracked by Altmetrics (Figure 1). Past or present authors of the SGEM HOP accounted for 54 of 111 tweets mentioned and 214,296 of 304,288 Twitter impressions during this period. Of note, the social media platforms for the American College of Emergency Physicians (@acepnow) and Society for Academic Emergency Medicine (@saemonline) accounted for 7,763 and 6,177 impressions, respectively, indicating positive bidirectional feedback between free open access medical education and traditional publications. The SGEM Facebook page contained seven posts on this topic and reached 8,564 readers.

\section{ONLINE DISCUSSION SUMMARY}

Discussants were directed to the SGEM blog for online discourse through the podcast, Facebook, and Twitter where conversations were active for seven days. The lead study author, Dr. Plint, posted in reply to questions and comments directed at her about the study. All 12 comments were included in the thematic analysis. Themes present in these posted conversations included the pathophysiology of bronchiolitis, variation in bronchiolitis management in different practice settings, and how chest $\mathrm{x}$-rays might cause clinicians to overuse antibiotics. Notably absent were critical commentary of study methodology and statistical analysis.

The comment by Andrew Tagg reminded readers of how prehospital treatments affected emergency department bronchiolitis management: "children [...] have often already received a dose of bronchodilator en route and so very few of our infants are likely to receive either further bronchodilators or steroids." He provides an anecdote on how a local quality improvement project facilitated knowledge translation in his own centre by decreasing 


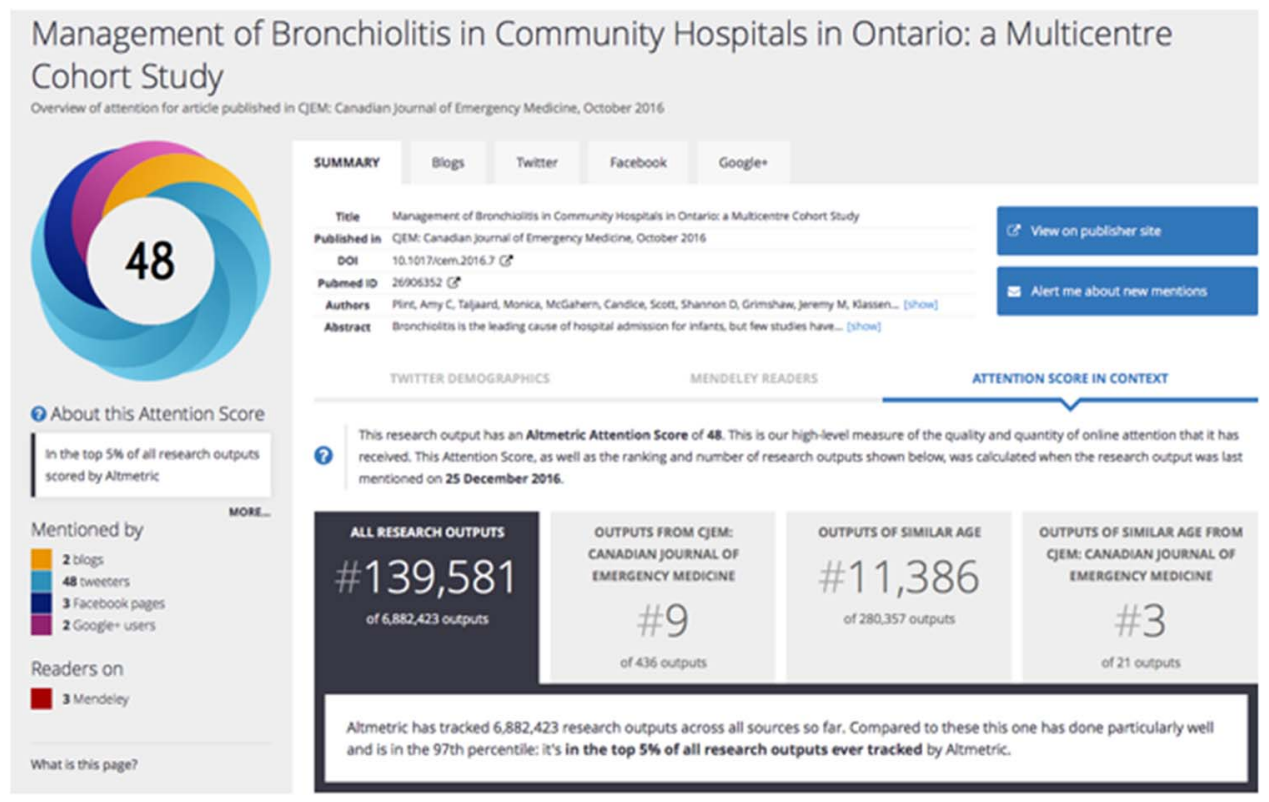

Figure 1. Screen capture of the Altmetrics data retrieved on December 25, 2016.

radiograph use. Dr. Tagg did not respond to our request for more information, but his reported experience aligns with previously published research demonstrating that institutional protocols and benchmarking might decrease $\mathrm{x}$-ray and bronchodilator use in these patients. 15,16

Tim Horeczko described bronchiolitis as "necrotic sludge and secretions [filling] lower airways and alveoli" that likely limits the role of any one treatment modality and highlighted his own preference for nebulized medications in patients whose first presentation of a fever, wheeze, and cough might exist along the reactive airway disease spectrum. This pathophysiologic explanation has been expressed by others in the past. ${ }^{17}$

\section{LIMITATIONS OF THE SOCIAL MEDIA ANALYSIS}

The limitations of this SGEM HOP summary include some of those addressed previously. ${ }^{18}$ The extent and quality of audience engagement could not be quantified through numerical indices such as Facebook reach statistics, Twitter impressions, and Altmetric score. Further, discussants on the SGEM blog are a self-selecting subset of the audience with an interest in FOAMed and knowledge translation. This point might have biased audience feedback in favour of supporting the authors' call for increased knowledge translation efforts in the community based on the results of this study. Publication of this SGEM HOP and online discussions occurred during winter holidays. This timing might have decreased viewership and participation. We also wonder whether the author's digital presence, as well as the generally positive evaluation of the study by the SGEM HOP, reduced methodological questions or critical commentary. However, such direct access to study investigators is rare and might eventually prove a valuable way of advancing scientific discourse similar to "Letters to the Editor." Perhaps future audienceauthor discussions on the SGEM HOP would benefit from a facilitator explicitly giving participants permission to ask difficult questions.

\section{CONCLUSIONS}

Plint and colleagues reported a descriptive analysis of bronchiolitis management in Canadian community EDs between 2005 and 2007. The SGEM podcast highlighted the disparity between modern treatment guidelines and practice patterns observed during the study period while reinforcing key concepts in bronchiolitis care during peak RSV season for much of the northern hemisphere. Subsequent online discussion revealed tension between guideline-based care and clinical practice realities even within the FOAMed setting, but discussants were generally supportive of increasing knowledge translation efforts to the community hospitals. 
Competing Interests: None declared.

\section{REFERENCES}

1. Thoma B, Mohindra R, Artz JD, Chan TM. CJEM and the changing landscape of medical education and knowledge translation. CFEM 2015;17(2):184-7.

2. Plint AC, Taljaard M, McGahern C, et al. Management of bronchiolitis in community hospitals in Ontario: a multicentre cohort study. CFEM 2016;8(6):443-52.

3. Hartling L, Fernandes RM, Bialy L, et al. Steroids and bronchodilators for acute bronchiolitis in the first two years of life: systematic review and meta-analysis. BMF 2011;342:d1714.

4. Garrison MM, Christakis DA, Harvey E, Cummings P, Davis RL. Systemic corticosteroids in infant bronchiolitis: a meta-analysis. Pediatrics 2000;105(4):E44.

5. Spurling GK, Doust J, Del Mar CB, Eriksson L. Antibiotics for bronchiolitis in children. Cochrane Database Syst Rev 2011;6:CD005189.

6. Mansbach JM, Emond JA, Camargo CA Jr. Bronchiolitis in US emergency departments 1992 to 2000: epidemiology and practice variation. Pediatr Emerg Care 2005;21(4):242-7.

7. Lieberthal AS, Bauchner H, Hall CB, et al. American Academy of Pediatrics Subcommittee on Diagnosis and Management of Bronchiolitis. Diagnosis and management of bronchiolitis. Pediatrics 2006;118(4):1774-93.

8. Andrade MA, Hoberman A, Glustein J, Paradise JL, Wald ER. Acute otitis media in children with bronchiolitis. Pediatrics 1998;101(4 Pt 1):617-9.

9. Bilavsky E, Shouval DS, Yarden-Bilavsky H, et al. A prospective study of the risk for serious bacterial infections in hospitalized febrile infants with or without bronchiolitis. Pediatr Infect Dis 7 2008;27(3):269-70.

10. Parikh K, Hall M, Teach SJ. Bronchiolitis management before and after the AAP guidelines. Pediatrics 2014;133(1): e1-7.

11. Van Cleve WC, Christakis DA. Unnecessary care for bronchiolitis decreases with increasing inpatient prevalence of bronchiolitis. Pediatrics 2011;128(5):e1106-12.

12. Facebook. What is the difference between impression and reach? 2016 https://www.facebook.com/help/2744003 62581037 (accessed December 28, 2016).

13. Symplur Analytics. Symplur analytics. Healthcare Hashtag Project. Available at: https://www.symplur.com/healthcarehashtags/sgemhop/ (accessed December 18, 2016).

14. Trueger NS, Thoma B, Hsu CH, et al. The Altmetric Score: a new measure for article-level dissemination and impact. Ann Emergency Med 2015;66(5):549-53.

15. Johnson LW, Robles J, Hudgins A, Osburn S, Martin D, Thompson A. Management of bronchiolitis in the emergency department: impact of evidence-based guidelines? Pediatrics 2013;131(Suppl 1):S103-9.

16. Ralston S, Garber M, Narang S, et al. Decreasing unnecessary utilization in acute bronchiolitis care: results from the value in inpatient pediatrics network. 7 Hosp Med 2013; $8(1): 25-30$.

17. Wright M, Mullett CJ, Piedimonte G. Pharmacological management of acute bronchiolitis. Ther Clin Risk Manag 2008;4(5):895-903.

18. Luckett-Gatopoulos S, Thoma B, Milne K, Bond C. SGEM Hot Off the Press: regional nerve blocks for hip and femoral neck fractures: a systematic review. CFEM 2016;18(4): 296-300. 